\title{
Renal Dendritic Cells Adopt a Pro-Inflammatory Phenotype in Obstructive Uropathy to Activate T Cells but Do Not Directly Contribute to Fibrosis
}

\author{
Sarah L. Snelgrove, ${ }^{*}$ Joshua Y. Kausman, ${ }^{*}$ \\ Cecilia Lo, ${ }^{*}$ Camden Lo, ${ }^{\dagger}$ Joshua D. Ooi, ${ }^{*}$ \\ P. Toby Coates, ${ }^{\ddagger}$ Michael J. Hickey, ${ }^{*}$ \\ Stephen R. Holdsworth, ${ }^{\star}$ Christian Kurts, ${ }^{\text {ๆ }}$ \\ Daniel R. Engel, ${ }^{\text {" }}$ and A. Richard Kitching ${ }^{\star \S \|}$
}

exhibit inflammatory morphological and functional characteristics and are more effective antigen-presenting cells, but they do not directly contribute to tubulointerstitial damage and fibrosis. (Am J Pathol 2012, 180:91-103; DOI: 10.1016/j.ajpath.2011.09.039)

Dendritic cells (DCs) are sentinels of the immune system, providing immunological surveillance and being capable of maintaining tolerance or initiating active immunity. These antigen-presenting cells (APCs) form heterogeneous populations with diversity in phenotype and function segregating subpopulations. Although different DC subsets and their relative functions have been extensively studied in secondary lymphoid organs, less is known about the phenotype and function of DCs in peripheral tissues such as the kidney. DCs constitute one of the major leukocyte populations in the kidney and are distributed in an organized and uniform fashion throughout the tubulointerstitium. ${ }^{1-4}$ Like conventional DCs, renal DCs express CD11c and major histocompatibility complex class II (MHC II) but some renal DCs express markers also associated with tissue macrophages (in the mouse CD11b, F4/80 and CX3CR1), making discrimination between the two subsets more difficult. ${ }^{3,4}$

Dendritic cells can act to promote or regulate renal disease in two ways. First, DCs have the capacity to act

This work was supported by grants from the National Health and Medical Research Council of Australia (Project Grant 565033), the Go8 AustraliaGermany DAAD Co-operation Scheme and the Deutsche Forschungsgemeinschaft grants SFBTR57 and KFO228.

Accepted for publication September 27, 2011.

A.R.K. and D.R.E. contributed equally to this work.

CME Disclosure: None of the authors disclosed any relevant financial relationships.

Supplemental material for this article can be found at http://ajp. amjpathol.org or at doi: 10.1016/j.ajpath.2011.09.039.

Address reprint requests to Richard Kitching, Ph.D., Centre for Inflammatory Diseases, Monash University Department of Medicine, Monash Medical Centre, 246 Clayton Road, Clayton, VIC 3168, Australia. E-mail: Richard.Kitching@monash.edu. 
as professional APCs to initiate or regulate antigen-specific (adaptive) immune responses by presenting antigen to $T$ cells. In addition to this role as a bridge between innate and adaptive immunity, DCs have the capacity to act locally as innate immune cells and to directly modify local inflammation. Although renal DCs have been shown to produce inflammatory mediators, for example tumor necrosis factor (TNF), ${ }^{5,6}$ few studies have investigated the functional role of DCs in renal disease (by the conditional ablation of DCs). In immune-mediated disease renal DCs have been reported as having early local antiinflammatory properties and later pro-inflammatory roles. $^{7,8}$ In cisplatin-induced acute kidney injury, a toxic nephropathy where there is no evidence for a role of adaptive immunity, DCs are protective via interleukin-10 production. $^{9}$

Clearly, DCs can produce pro-inflammatory and anti-inflammatory cytokines, which may exacerbate or ameliorate pathological processes. DCs have been implicated in a variety of important pathological conditions, and this article defines their contribution to the early inflammatory events in renal fibrosis. Renal fibrosis occurs as a consequence of chronic kidney disease in both children and adults, and the consequent progressive decline in kidney function may ultimately lead to renal failure. Unilateral ureteric obstruction (UUO) is a common experimental model used to study renal fibrosis. Obstruction initiates a series of pathological changes, including interstitial inflammation, pro-inflammatory cytokine release, tubular cell apoptosis, myofibroblast activation, and tubulointerstitial fibrosis, all of which have deleterious effects on kidney function. ${ }^{10,11}$ DCs are present in the kidney and early after UUO, secrete proinflammatory cytokines. ${ }^{6} \mathrm{Al}$ though some, ${ }^{12}$ but not other studies ${ }^{13}$ suggest a role for T cells in obstructive uropathy, there is no documented role for antigen-specific responses. However antigen-specific responses, including those relating to infection and to allogeneic responses, can occur in the presence of obstruction in native and transplant kidneys.

The present study uses a range of techniques, including DC-specific tools, CD11c-promoter driven enhanced yellow fluorescent protein (EYFP) reporter, and diphtheria toxin receptor (DTR) depletion mice, to define the role of DCs in the kidney, using UUO as a model inflammatory disease. By means of in vivo multiphoton microscopy and ex vivo phenotypic analyses, this study provides an analysis of both renal DCs from healthy kidneys and the changes induced in this population after obstruction. Renal DCs undergo morphological changes and are functionally activated in the injured kidney, consistent with a pro-inflammatory phenotype. The low capacity of renal DCs (compared with splenic DCs) to induce T-cell proliferation is increased in the inflammatory milieu that exists after UUO. Depletion studies show that although renal CD11c+ cells become activated during inflammation (assessed by surface phenotype, cytokine production and antigenpresenting capacity), renal DCs do not directly contrib- ute to the progression of apoptosis or fibrosis in the kidney in the early stages after UUO.

\section{Materials and Methods}

\section{Mice and Unilateral Ureteric Obstruction}

C57BL/6 and OT-I mice [TCR transgenic specific for $\mathrm{H}-2 \mathrm{~K}^{\mathrm{b}}$ and ovalbumin (OVA $\left.{ }^{14}\right]$ were bred at Monash Animal Services (Melbourne, Australia). CD11C-EYFP ${ }^{15}$ and rat insulin promoter (RIP)-mOVA mice expressing membrane-bound OVA under the control of the rat insulin promoter ${ }^{16}$ were bred and maintained at Monash Medical Centre (Melbourne, Australia). CD11cDTR mice, ${ }^{17}$ which also express green fluorescent protein (GFP) under the control of the CD11c promoter, were bred at the Institute for Molecular Medicine and Experimental Immunology (Bonn, Germany). All mice were on a C57BL/6 background. Experiments were conducted in mice 8 to 14 weeks of age and were approved by the Monash Medical Centre Animal Ethics Committee or the German Institutional and Government Review Boards.

Adult male mice were anesthetized via inhalation of sevoflurane (Abbotts Laboratories Ltd, Berkshire, UK). UUO was performed through a midline incision. After the left ureter was identified, it was tied with silk suture at two points and permanently ligated. Sham-operated mice underwent an identical procedure but without ureteric ligation. At 3,7 , or 14 days postobstruction, mice were sacrificed and kidneys collected. For DC depletion, sexmatched, adult CD11C-DTR transgenic mice were used. CD11c-DTR transgenic or control (transgene negative or wild-type) mice were injected i.p with $4 \mathrm{ng}$ of diphtheria toxin (DT; Sigma-Aldrich, St. Louis, MO) per gram of body weight to specifically deplete CD11c+ DCs, as previously described. The administration of DT to the control mice does not deplete DCs. Depletion of renal CD11c+ cells in CD11c-DTR mice, measured 2 days after administration of DTR, as $72 \%$ (means of $n=2$ depleted and $n=$ 2 nondepleted mice), consistent with the degree of depletion of renal CD11c + cells using the CD11c-DTR in previous studies. ${ }^{7-9}$ Depletion was performed on days 0,2 , 4, or 7. On day 7 (day 14 for mice depleted at day 7), mice were sacrificed and the kidneys collected for histological analysis. Kidney samples were fixed in $2 \%$ paraformaldehyde, embedded in paraffin, and sectioned at $4 \mu \mathrm{m}$.

\section{Renal Multiphoton Microscopy}

Intravital multiphoton microscopy of the intact kidney was performed on mice anesthetized by i.p. injection of $150 \mathrm{mg} / \mathrm{kg}$ ketamine hydrochloride and $10 \mathrm{mg} / \mathrm{kg} \mathrm{xy}$ lazine, as described previously. ${ }^{18}$ The jugular vein was cannulated for the administration of fluorescent reagents and additional anesthetic. The left kidney was exteriorized through a lateral skin incision and held in a kidney holder specifically molded to the shape of the left kidney. Surgical glue was used to aid in keeping the kidney in place. Mice were kept at a constant temperature of $37^{\circ} \mathrm{C}$ on a heating pad. The kidney was 
immersed in bicarbonate buffered saline at $37^{\circ} \mathrm{C}$ and covered with a coverslip.

Time lapse recordings of EYFP+ DCs in the kidney of CD11c-EYFP mice were captured using a Leica SP5 mulitphoton microscope system, equipped with a $20 \times$ 1.0 NA water immersion objective (Leica Microsystems, Mannheim, Germany). Fluorophores were excited by a SpectraPhysics MaiTai pulsed infrared laser tuned to $860 \mathrm{~nm}$, with emissions captured by nondescanned detectors with 525/50 nm (YFP), 590/50 nm (TRITC), and 650/50 nm (Alexa 633) filters. Images were collected with $\sim 120 \mu \mathrm{m}$ Z-depth in 6- $\mu \mathrm{m}$ steps, with one Z-stack every 30 s for 15 to 25 minutes. Images were processed using Imaris (v7.0, Bitplane AG), and analysis was performed in Microsoft Excel (Microsoft Corporation, Redmond, WA). Specificity of EYFP expression by renal DCs in these reporter mice was confirmed by flow cytometry, where at least $95 \%$ of all EYFP+ cells expressed CD11c (see Supplemental Figure S1 at $h$ ttp://ajp.amjpathol.org).

To determine DC activity, in vivo changes in morphology were tracked for individual DCs and a morphology index derived. Each DC three-dimensional shape can be described by the following mathematical model of sphericity, ${ }^{19}$ where the value is between 0 (an absolute 2D object) and 1 (a perfect sphere): Sphericity $=\pi^{1 / 3}(6 \times$ cell volume $)^{2} / 3 /$ cell surface area. The sphericity of each DC was then tracked over time, with the sphericity difference between consecutive time points averaged over the entire time course to generate a value which describes the activity of DCs, as described by the rate of change in their morphology index $(\Delta \mathrm{Ml})$. Cells with relatively low $\Delta \mathrm{Ml}$ values change morphology less than cells with relatively high $\Delta \mathrm{Ml}$ values.

\section{Isolation of DCs from Kidney or Lymph Nodes}

Kidneys and lymph nodes (LN) were digested with 1 $\mathrm{mg} / \mathrm{mL}$ Collagenase $\mathrm{D}$ and $0.1 \mathrm{mg} / \mathrm{mL}$ DNase I (both Roche Diagnostics, Indianapolis, IN), as described previously. ${ }^{3}$ After washing in RPMI $1640 / 10 \%$ fetal calf serum, kidney preparations were left to settle for 10 minutes to remove tubular debris. Erythrocytes were lysed with ammonium chloride and single cell preparations filtered through 70- $\mu \mathrm{m}$ mesh. In some experiments, DCs were further sorted by flow cytometry (MoFlo XDP, Beckman Coulter, Miami, FL) by sorting on the CD45+CD11c+ population. Postsorting analysis of sorted DCs showed the purity was $>90 \%$.

\section{Flow Cytometry}

Cells were preincubated with anti-CD16/CD32 antibody (Ab) for 10 minutes to block nonspecific staining, then stained with directly conjugated primary $A b$ at predetermined optimal concentrations for 30 minutes. Fluorescentl labeled monoclonal $\mathrm{Ab}$ used were against CD45 (30-F11), CD11c (HL3), CD11b (M1/70), F4/80, MHC II (M5/114.15.2), Gr-1 (RB6-8C5), B220 (RA36B2), CD19 (1D3), CD3 (17A2), CD4 (GK1.5), CD8 (53-6.7), V $\alpha 2$ (B20.1), TNF (MP6-XT22), or IL-12p40
(C15.6). Dead cells were excluded by staining with propidium iodide. To assess cytokine production by DCs, single cell kidney suspensions were incubated in $1 \mathrm{~mL} \mathrm{RPMl} / 10 \% \mathrm{FCS}$ with $10 \mu \mathrm{g} / \mathrm{mL}$ Brefeldin A (4 hours at $37^{\circ} \mathrm{C}$ ) before staining with surface markers. Cells were fixed and permeablized using the Cytofix/Cytoperm kit (BD Pharmingen, San Diego, CA) then stained intracellularly with anti-cytokine antibodies for 20 minutes. To enumerate the total number of cells, a known number of BD Calibrate Beads (BD Biosciences, San Jose, CA) was added per sample. Cells were acquired on a FACSCanto flow cytometer (BD Biosciences) and analyzed with FlowJo software (Tree Star, Ashland, OR). Stringent fluorescence minus one controls of both normal and obstructed kidney samples were used to ensure specificity in data analysis.

\section{T-Cell Isolation, ex Vivo T-Cell Stimulation, and in Vivo T-Cell Proliferation Assays}

CD8 $T$ cells from naive OT-I donors were positively enriched from the spleen and LN by MACS (MiltenyiBiotec, Auburn, CA) using anti-CD $8 \alpha$ conjugated to microbeads. Enriched CD8 T cells were resuspended $\left(1 \times 10^{7}\right.$ cells $\left./ \mathrm{mL}\right)$, labeled with $5 \mu \mathrm{mol} / \mathrm{L}$ carboxyfluorescein succinimidyl ester (CFSE), (Molecular Probes, Eugene, OR) then washed twice. A total quantity of $1 \times$ $10^{5}$ enriched CFSE-labeled OT-I CD8+ TCR transgenic cells was added to titrated numbers of sorted DCs that were pulsed with $\mathrm{OVA}_{257-264}$ peptide. Cells were cultured in a final volume of $200 \mathrm{~mL}$ RPMI 1640 containing $10 \%$ fetal calf serum, $50 \mathrm{mmol} / \mathrm{L} 2-\mathrm{ME}, 2$ $\mathrm{mmol} / \mathrm{L}$ L-glutamine, $100 \mathrm{U} / \mathrm{mL}$ penicillin, and 100 $\mathrm{mg} / \mathrm{mL}$ streptomycin in 96-well, U-bottomed plates. Cells were harvested after 60 hours and were stained with antibody, and proliferation was analyzed by flow cytometry.

To assess DC function in vivo, whole-single-cell lymphocyte suspensions from pooled OT-I spleen and LN were CFSE labeled and adult male RIPmOVA mice were i.v. injected with $10 \times 10^{6} \mathrm{CFSE}-l a b e l e d$ lymphocytes (equating to $3.5 \times 10^{6}$ OT-I T cells). The left ureter of recipient mice was surgically ligated or control mice underwent sham operation. At day 3 after surgery, mice were sacrificed, and the renal LNs [UUO and contralateral $(C L)]$ were collected. Cells were stained and then analyzed by flow cytometry as described above.

\section{Histology and Immunohistology}

Kidney sections were stained with Picrosirius red stain, and histological assessment of collagen deposition was determined using polarized light microscopy. Nonoverlapping images of the entire kidney section were taken using Nikon Abrio Polarized light microscope (×200 magnification). National Institutes of Health $(\mathrm{NIH})$ Image J software was used to analyze the images and results were expressed as percentage of total area. Interstitial myofibroblast [ $\alpha$-smooth muscle actin $(\alpha$-SMA)], cleaved 
caspase-3 expression, CD4 ${ }^{+} \mathrm{T}$ cells, and CD8+ T cells were assessed by immunohistochemistry. For $\alpha$-SMA and cleaved caspase-3, paraffin-fixed sections were stained with mouse anti-human $\alpha$-SMA monoclonal Ab (Dako, Victoria, Australia) or polyclonal rabbit anti-mouse cleaved caspase-3 Ab (Asp175, Cell Signaling Technology, Danvers, MA) overnight at $4^{\circ} \mathrm{C}$. For T cells, $6-\mu \mathrm{m}-$ thick frozen kidney sections were air dried and acetone fixed, and blocked with 10\% rabbit serum before incubation overnight at $4^{\circ} \mathrm{C}$ with either rat anti-mouse CD4 (GK1.5, American Type Culture Collection [ATCC], Manassas, VA, $40 \mu \mathrm{g} / \mathrm{mL}$ ) or CD8 (53-6.7, ATCC, $40 \mu \mathrm{g} /$ $\mathrm{mL}$; rat IgG2a and IgG2b were isotype controls). Secondary steps were, for $\alpha$-SMA, rabbit anti-mouse immunoglobulin horseradish peroxidase (Dako), for cleaved caspase-3, Signal Stain Boost IHC detection reagent (Cell Signaling), and for T cells, rabbit anti-rat biotinylated immunoglobulin, followed by incubation with Vectastain $\mathrm{ABC}$ reagent (Vector Laboratories, Burlingame, CA). Bound antibodies were detected with 3,3'diaminobenzidine (Sigma-Aldrich) and sections were counterstained with $1 \%$ nuclear fast red. For $\alpha$-SMA, 5-10 nonoverlapping high-power $(\times 400)$ fields (HPF) of each kidney section were captured and quantified using Metamorph analysis software (Molecular Devices, Downingtown, PA) and positively stained area expressed as percentage of total area. For cleaved caspase-3 and T cells, the number of positive cells was counted in 10 to 15 high-power fields. Sections stained with Periodic AcidSchiff were assessed for tubular injury, including tubular dilation, cast formation, tubular atrophy and the presence of inflammatory infiltrate. To confirm the findings for cleaved caspase-3, apoptotic cells in kidneys in some experiments were identified using the ApopTag In Situ Apoptosis Detection Kit (Millipore-Chemicon, CA), using 2- $\mu \mathrm{m}$ paraformaldehyde-fixed paraffin sections according to the manufacturer's instructions. In addition, 3,3'diaminobenzidine was used to reveal numbers of the tubular and interstitial TdT-dUTP nick end labeling (TUNEL)-positive apoptotic cells in the cortex, assessed in at least 10 high-power fields.

\section{Statistical Analysis}

All values are expressed as mean \pm SEM. Statistical analysis was performed using the unpaired $t$-test (GraphPad Prism 5; GraphPad Software Inc., San Diego, CA). A $P$ value of less than 0.05 was considered significant. In all figures, ${ }^{\star} P<0.05,{ }^{* *} P<0.01$, and ${ }^{\star \star \star} P<0.001$. The number of repeat experiments to ensure reproducibility of results is reported in the figure legends.

\section{Results}

\section{Dendritic Cells Undergo Morphological Changes After Ureteric Obstruction}

To determine the behavior of renal DCs in normal and injured kidneys, kidneys of live CD11cEYFP reporter mice were exteriorized and the DCs were visualized by multiphoton microscopy. DCs were present within the cortical interstitium forming a continuous, interdigitating network (Figure 1A, concordant with previous confocal images of CX3CR1-GFP+ cells in the normal kidney ${ }^{4}$ ). Some DCs showed actively probing dendrites; however, in contrast to dermal DCs, ${ }^{20}$ renal DCs were essentially immotile (Figure 1C; see also Supplemental Video S1 at http://ajp.amjpathol.org). Changes in DC morphology and activity were further investigated in inflammation. Three days after UUO (d3 UUO), the highly structured arrangement of DCs seen in normal mice was disrupted with DCs clustering around damaged (more autofluorescent) tubules (Figure 1B). Similar findings were seen in paraffinfixed kidney sections (day 7 after UUO; see Supplemental Figure S2 at http://ajp.amjpathol.org). The morphology of the DCs present in the kidney had changed by day 3 after obstruction, becoming more stellate than in control mice (Figure 1, C and D). Individual EYFP+ DCs were further assessed for morphological changes over time. In vivo observations showed that DCs in the injured kidney exhibited more extension and retraction of dendrites in the renal interstitium compared with control renal DCs (Figure 1, C-E; see also Supplemental Videos S1 and S2 at $h$ ttp://ajp.amjpathol.org). Some mobile DCs were also consistently identified adjacent to damaged tubules after UUO, such mobility was rarely seen in the normal kidney (see Supplemental Videos S1 and S2 at http://ajp. amjpathol.org). Between normal and injured kidneys, absolute numbers of DCs were similar and the length of dendrites on DCs remained unchanged (mean dendrite length $10 \mu \mathrm{m}$; Figure 1, F and G). However, the number of dendrite extensions per DC was doubled in kidneys 3 days after UUO (Figure $1 \mathrm{H}$ ). These are the first in vivo microscopy studies of renal DCs in disease and provide evidence of in vivo DC activation in obstructed kidneys.

\section{Ureteric Obstruction Promotes Leukocyte Recruitment}

At 3 days after UUO, leukocytes within the kidney were analyzed by flow cytometry and compared with kidneys from sham-operated mice. Leukocytes, isolated by enzymatic and mechanical digestion steps, were incubated with the pan-leukocyte marker, CD45 and a range of specific cell surface markers to determine the proportion and number of $\mathrm{CD}^{+}$and $\mathrm{CD} 4^{+}$cells, DCs, $B$ cells, macrophages, monocytes, and neutrophils. The proportions and cell number of monocytes and neutrophils were significantly increased in obstructed kidneys, accompanied by an increase in CD8 T cells (Figure 2, A and B), consistent with previous findings. ${ }^{6}$ Although the proportion of DCs and macrophages (of all $\mathrm{CD}_{4} 5^{+}$cells) was lower in injured kidneys, reflecting the influx of other leukocyte populations to the site of inflammation, actual total numbers of both DCs and macrophages were unchanged compared with kidneys from sham-operated mice. 

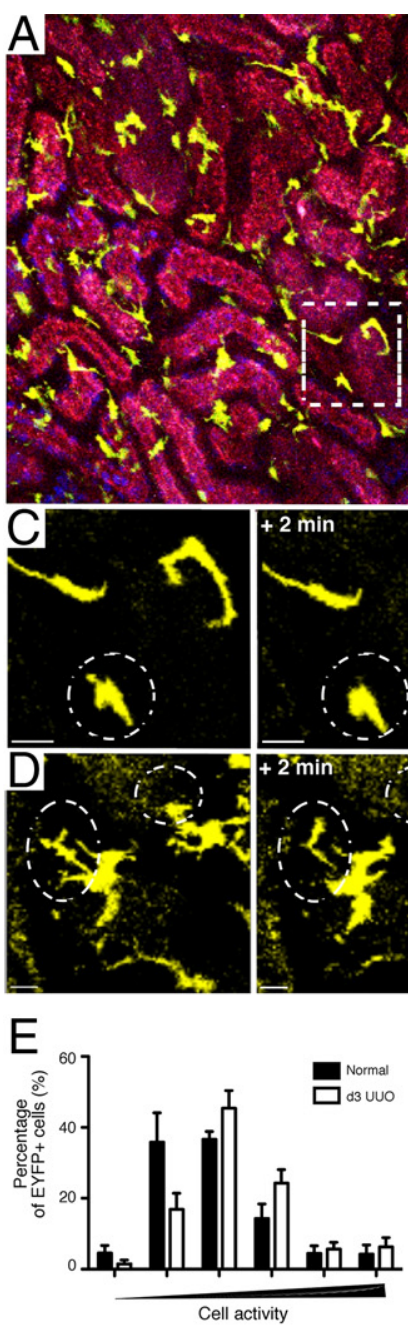

\section{Phenotype of DC Subsets in Kidney Is Altered After Ureteric Obstruction}

Renal DCs are heterogeneous, consisting of several subsets, distinguishable by their cell surface marker expression. To characterize the phenotype of the DC subsets in the kidney during inflammation, single-cell kidney suspensions from individual mice 3 days after UUO, or shamoperated controls, were analyzed by multicolor flow cytometry. Gating on $\mathrm{CD}_{4} 5^{+} \mathrm{CD} 11 \mathrm{c}^{+} \mathrm{DCs}$, the expression of other cell surface markers were assessed (Figure 3A). In control mice, the majority of renal DCs expressed F4/80 and $\mathrm{CD} 11 \mathrm{~b}$ as previously described. ${ }^{3} \mathrm{DC}$ s isolated from mice 3 days after UUO exhibited different surface expression of these markers, with an increase in the both proportion and absolute number of $\mathrm{F} 4 / 80^{-} \mathrm{DCs}$, coinciding with fewer $\mathrm{F} 4 /$ $80^{+}$DCs (Figure 3, A and B). A reduction in resident DCs that were $\mathrm{MHCI}{ }^{+} \mathrm{F} 4 / 80^{+}$was accompanied by an increase in the $\mathrm{MHCll}^{+} \mathrm{F} 4 / 80^{-}$subset (Figure $3 \mathrm{~B}$ ). As F4/80 expression is reportedly decreased on antigen uptake and DC maturation, ${ }^{21}$ this finding suggests that some renal DCs are exhibiting a more mature phenotype. The level of CD11b expression was increased (Figure $3, A$ and $C$ ), and a subset of DCs expressing high levels of CD11b and Gr-1 was
Figure 1. DCs undergo morphological changes during early inflammatory events after UUO. Renal DCs were imaged using multiphoton microscopy, and DCs were assessed in the kidneys of normal mice (not operated on, $n=5$ ) and at 3 days after UUO $(n=5)$. A representative maximum projection image (three-dimensional image $\mathrm{x}, \mathrm{y}$, and $\mathrm{z}$, compressed into two dimensions, $\mathrm{x}$ and $\mathrm{y}$ ) is shown for normal DCs (A) and at day 3 after UUO (B). DCs expressing EYFP are shown in yellow; the tubular epithelial cells are highlighted in red by the incorporation of a cell tracker dye; and Hoechst nuclear stain is shown in blue. Magnification of the boxed areas in panels $\mathbf{A}$ and $\mathbf{B}$ are shown as time lapse panels at four time intervals, each 2 minutes apart for normal DCs $(\mathbf{C})$ and d3 UUO (D). Dotted circles encompass dendrites that exhibit morphological changes over time. The movement or activity of each individual DC was measured by the change in the morphology index over time $(n=$ 4/group). The percentage of DCs exhibiting increasing levels of cellular activity is shown (E), as is the number of DCs per field of view $(\mathbf{F})$, the estimated length of the dendrites on the DCs $(\mathbf{G})$, and the mean number of dendrites per DC $(\mathbf{H})$, ${ }^{*} P<0.05$. found almost exclusively in the obstructed kidneys (Figure $3, \mathrm{~A}$ and $\mathrm{C}$ ). An increase in the number of immature

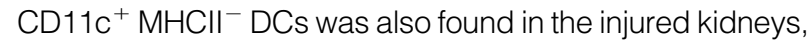
consistent with this subset being a DC precursor population. ${ }^{3,22}$ Proportions of $\mathrm{B}^{2} 2 \mathrm{O}^{+}$plasmacytoid DCs (pDCs) and $\mathrm{CD} 8 \alpha^{+}$conventional DCs were also increased, with an increased absolute number of pDCs, but not CD8 $\alpha^{+}$DCs (Figure 3, A and D). Thus, in UUO-induced inflammation, renal DCs undergo phenotypic changes consistent with an increased level of maturation of DCs in the kidney.

Cytokine production by renal DCs, specifically the production of TNF and IL-12p40 (the common chain for IL-12 and IL-23) was assessed by intracellular flow cytometry, comparing kidneys from mice 3 days after UUO with shamoperated mice. The proportion of $\mathrm{CD}_{11 \mathrm{c}^{+}}$cells producing either TNF or IL-12p40 was not altered by UUO (Figure 4, A and $\mathrm{B}$ ). However, more $\mathrm{CD} 11 \mathrm{C}^{+} \mathrm{F} 4 / 80^{-}$cells made TNF and IL-12p40 after UUO (though for IL-12p40, proportions were still low). TNF production by $\mathrm{CD} 11 \mathrm{c}^{+} \mathrm{CD} 11 \mathrm{~b}^{\mathrm{int}}$ cells was similar with and without UUO: a higher proportion of CD11c+ cells with hi or lo CD11b expression made TNF (Figure 4C). Fewer CD11c ${ }^{+}$CD11 bo cells made IL-12p40, although proportions, even in sham-operated mice, were very low (Figure 4D). 

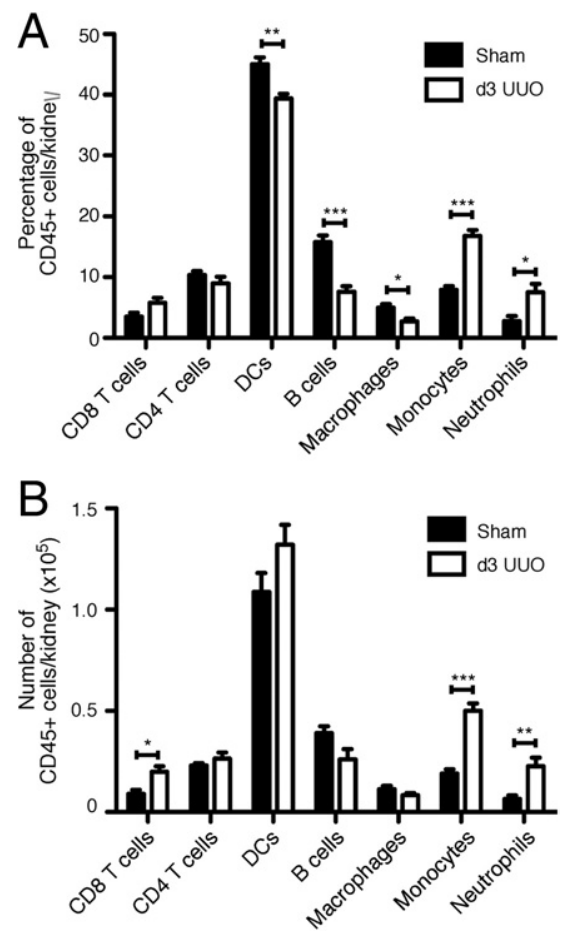

Figure 2. Increased numbers of CD8 T cells, monocytes, and neutrophils are recruited to the injured kidney whereas the total numbers of renal DCs are unchanged. Renal CD45+ leukocyte populations were isolated from whole kidneys from mice at $\mathrm{d} 3$ UUO or subjected to sham operation. Cell populations were assessed by multicolor flow cytometric analysis. Staining definitions for the individual cell populations were CD8 T cells: $\mathrm{CD} 45^{+} / \mathrm{CD}^{+} /$ $\mathrm{CD}^{+}$. CD 4 T cells: $\mathrm{CD}_{4} 5^{+} / \mathrm{CD}^{+} / \mathrm{CD}^{+}$. DCs: $\mathrm{CD} 45^{+} / \mathrm{CD} 11 \mathrm{c}^{+}$. B cells:

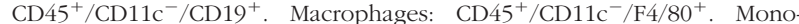

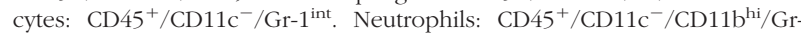
1 hi. The proportions (A) and absolute cell numbers (B) of individual leukocyte subsets are shown. Each cell population is expressed as the mean \pm SEM of five mice per group. Similar results were reproduced in three experiments. ${ }^{*} P<0.05$; *** $P<0.01 ;{ }^{* * * *} P<0.001$

\section{Level of CD11c Expression Relates to} Differential Expression of Markers Overlapping with Monocyte/Macrophage Lineage

Monocytes can differentiate into DCs in both steady-state and inflammatory conditions. ${ }^{23-25}$ Changes in DC subsets may be attributed to the recruitment of monocytic DC precursors with subsequent differentiation into DCs, activation of resident renal DCs, or both. Typically, the closer to a monocyte precursor a cell is, the lower the CD11c expression. ${ }^{26}$ To address this issue, cells that expressed CD11c were divided into CD11c low, intermediate, or high and analyzed for the expression of markers that overlap with the monocyte/macrophage lineage (MHC II, Gr-1, F4/80 and CD11b). At day 3 after obstruction there was a threefold increase in the proportion of DCs that were CD11c high (Figure 5A). The alterations in MHC II, Gr-1, F4/80, and CD11b expression postobstruction, outlined in Figure 3, related to the degree of $\mathrm{CD} 11 \mathrm{c}$ expression. With increasing levels of CD11c, F4/80 expression decreased and CD11b expression increased. However, MHC II $^{\mathrm{o}}$ cells, and $\mathrm{Gr}-1^{+}$ cells were more often expressing low to intermediate levels of CD11c (Figure $5 \mathrm{C}$ ). When comparing changes in the damaged kidney to the sham-operated mice, CD11c high DCs were of similar phenotype in both groups (although more of this subset was present in the inflamed kidney). The differences in phenotype between sham-operated and obstructed kidneys were largely due to changes in cells expressing lower levels of CD11c. These results indicate that resident renal cells undergo maturation changes in the obstructed kidneys involving the loss of F4/80 expression and an increased level of CD11b. DCs migrate from inflamed tissue to regional $\mathrm{LNs}^{27}$ and more DCs were present in the draining renal $L N$ of obstructed kidneys compared with sham operated kidneys (Figure 5D). As the absolute numbers of DCs in the kidney were similar between sham operated and d3 UUO mice (Figure $2 \mathrm{~B}$ ), this efflux of resident DCs must be accompanied by recruitment into the kidney. The phenotype of immature MHC $\|^{10}$, monocyte-derived inflammatory cells with lower levels of CD11c is typical of recruited inflammatory APCs and consistent with these cells as recent immigrants to the obstructed kidney.

\section{Reduced Capacity of Renal DCs to Stimulate $T$-Cell Responses Is Increased After UUO}

Given the selective increase of $\mathrm{CD} 11 \mathrm{c}^{\text {hi }}$ cells in the kidney and the renal LN after UUO, the antigen-presenting capacity of these cells was assessed ex vivo as a measure of their ability to stimulate antigen-specific CD8 T-cell responses. Purified syngeneic DCs, isolated from either spleen or kidney, were pulsed with $\mathrm{OVA}_{257-264}$ peptide before culture with naive, CFSE-labeled, OVA-specific OT-I T cells. Flowcytometric analyses using serial CFSE dilution showed that renal DCs from untreated kidneys were less capable of stimulating antigen-specific CD8 T-cell responses than splenic DCs (Figure 6A). The majority of renal DCs are $\mathrm{F} 4 / 80+$ (unlike splenic DCs), and it has been suggested that the $\mathrm{F} 4 / 80$ molecule plays a role in tolerance induction. ${ }^{28}$ Spleen and kidney DCs were mixed to determine whether renal DCs diminished the capacity of splenic DCs to induce OT-I proliferation. However, when stimulated by equal numbers of splenic and renal DCs (500 splenic and 500 renal DCs per culture), numbers of proliferating OT-I cells were not reduced compared with cells stimulated by 500 splenic DCs alone (Figure 6B). Similar results were obtained when using splenic and renal DCs at several other ratios (data not shown).

Using UUO as a model inflammatory condition within the kidney, we assessed the classical function of DCs, that is, their capacity to stimulate T-cell responses. This function was enhanced in renal DCs 3 days after UUO, with the proportion of proliferating OT-I cells and the number of cells undergoing one to seven divisions being similar to that recorded for splenic DC stimulated T cells (Figure 6, C and D). In vivo T-cell proliferation assays were conducted using RIP-mOVA transgenic mice that express membrane-bound OVA on kidney proximal tubule cells. ${ }^{16}$ RIPmOVA mice received CFSE-labeled OT-I CD8 T cells followed by surgical UUO or underwent sham operation. Three days postoperatively, the draining renal $L N s$ (UUO and $\mathrm{CL}$ ) were collected for flow-cytometric analyses (Figure 6E). The number of OVA-specific CD8 T cells was sevenfold higher in the UUO renal $L N 3$ days postoperatively compared with the $\mathrm{CL}$ renal LN or the draining UUO LN in sham-operated mice 
A
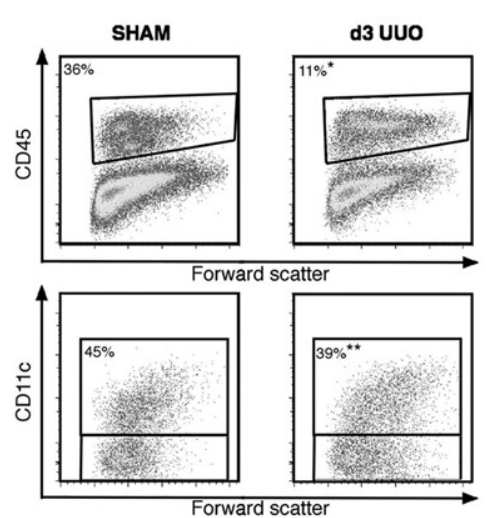

$\mathrm{B}$

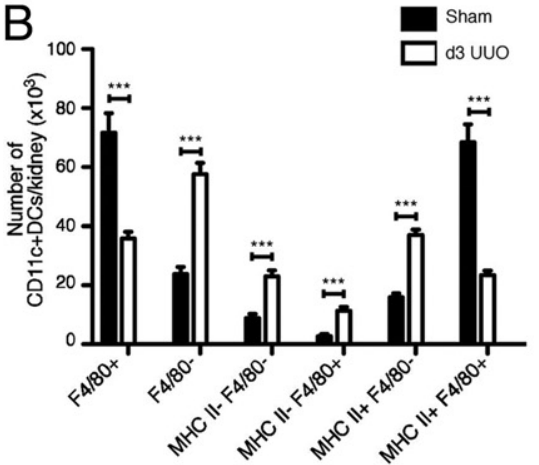

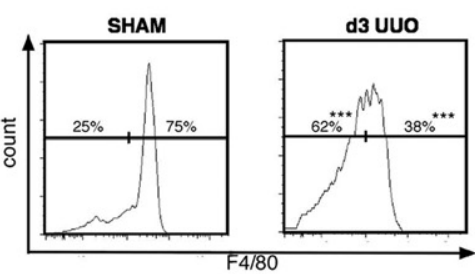
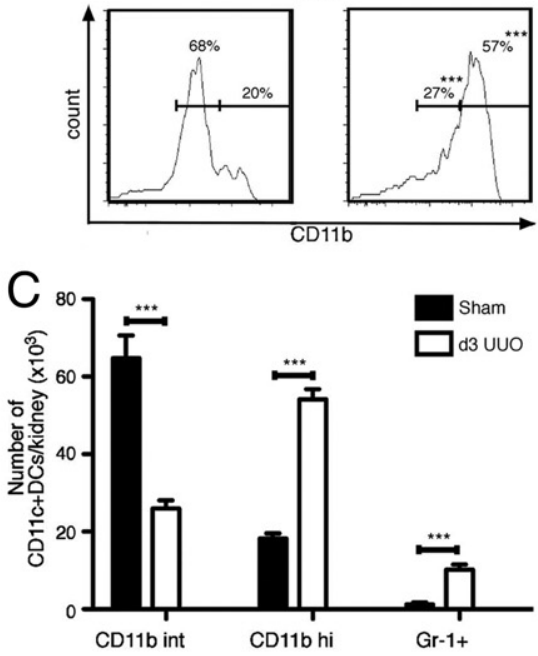
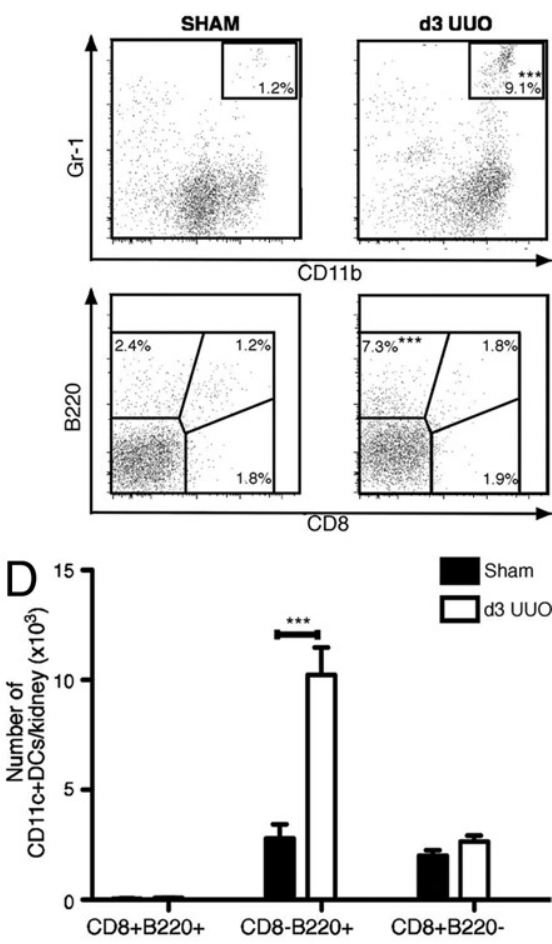

Figure 3. Renal DCs undergo phenotypic changes after ureteric obstruction. Renal DC subsets were assessed by multicolor flow-cytometric analysis of single-cell kidney suspensions from mice at d3 UUO or sham operated controls ( $n=5 /$ group). A: Representative dot plots indicate the gating strategy used to identify DCs by the expression of CD 45 and the DC specific marker, CD11c. DC subsets were further assessed for the expression of F4/80, CD11b, Gr-1 versus CD11b and B220 versus CD8a. Numbers indicate the mean percentage of DCs in the given subset. B-D: The absolute number of DCs categorized by the designated DC subsets is shown. Similar results were obtained in three individual experiments. ${ }^{* * *} P<0.01 ;{ }^{* * * * *} P<0.001$.

(Figure 6F), confirming that renal DCs are functionally activated after UUO.

\section{Conditional Ablation of DCs Does Not Hinder Progression of Fibrosis in Obstructed Kidneys}

To determine whether DCs play a direct role in innate renal inflammation leading to fibrosis DCs were conditionally ablated during UUO. In CD11cDTR mice, DCs repopulate the kidney 3 days after DT. ${ }^{7,9}$ In separate experiments, DT was administered to CD11cDTR mice and controls (transgene negative littermates and wild-type mice) on day 0,2 , or 4 after UUO, and renal tissue was assessed histologically on day 7 (Figure 7A). Depleting DCs at day 0 (on the day of surgery), day 2 , or day 4 allowed assessment of the roles of resting tissue DCs and of more recent immigrants. By day 7 , obstructed kidneys displayed tubulointerstitial damage, with dilated tubules, cast formation, tubular atrophy, and inflammatory infiltrates (Figure 7, B and C). Collagen was deposited in the interstitium, tubular cell apoptosis was present and $\alpha$-SMA+ cells (a marker for myofibroblasts) were accumulating, hallmarks in the development of renal fibrosis (Figure 7, D and H; Figure 8, A and E; and Figure 9, $A$ and $E$ ). The selective depletion of DCs at any time point, initially after UUO on day 0 , at an intermediate time of day 2 or in the later stages of obstruction at day 4 , had no significant impact on the extent of collagen deposition in the renal cortex (Figure 7). Depleting DCs (at day 0: $n=5$ mice not
DC depleted, $n=6$ mice DC depleted) did not significantly alter the early renal interstitial infiltration (assessed at day 4) of $\mathrm{CD}^{+}$cells (UUO with DCs $2.1 \pm 0.3$, UUO DC depleted $1.4 \pm 0.4$ cells/HPF, $P=0.15$ ) or CD8+ cells (UUO with DCs $1.0 \pm 0.3$, UUO DC depleted $0.7 \pm 0.2$ cells/HPF, $P=$ $0.35)$.

Tubular apoptosis occurs before the onset of frank fibrosis. In models of ischemia/reperfusion injury and UUO, including in the current study, renal DCs produce TNF, a cytokine that can induce apoptosis. ${ }^{5,6}$ Therefore, paraffinembedded sections were stained for cleaved caspase-3, a marker of apoptosis. Similar to the results obtained for collagen deposition, mice that were depleted of DCs at day 0 , 2 , or 4 during UUO showed no significant difference in tubular apoptosis compared with nondepleted control mice (Figure 8). TUNEL staining performed on sections from non-DC-depleted mice and DC-depleted mice at day 0 also showed no difference in apoptosis (UUO with DCs 1.0 \pm 0.1 , UUO DC day 0 depleted $0.9 \pm 0.2$ cells/HPF). Myofibroblast accumulation, important in renal fibrosis, can be defined by the expression of $\alpha$-SMA. Although, in normal kidneys, $\alpha$-SMA expression is restricted to the vessels, in renal fibrosis expression extends to the tubulointerstitium. The depletion of DCs at this series of time points showed that, compared with control mice given DT, there was no difference in the expression of $\alpha$-SMA in mice depleted of DCs at day 0, 2, or 4 (Figure 9). Collectively, the histological analysis of these three hallmarks of injury in this model- 


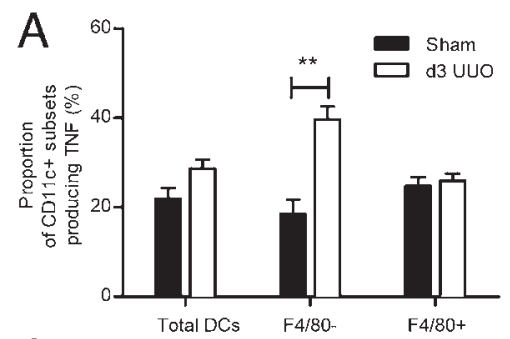

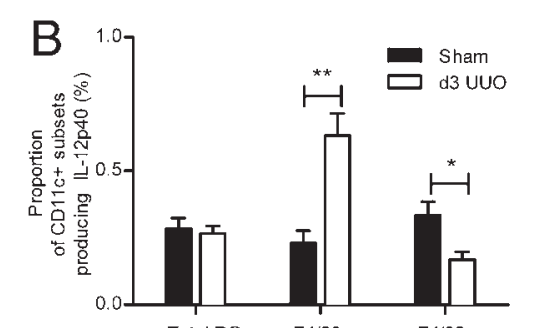

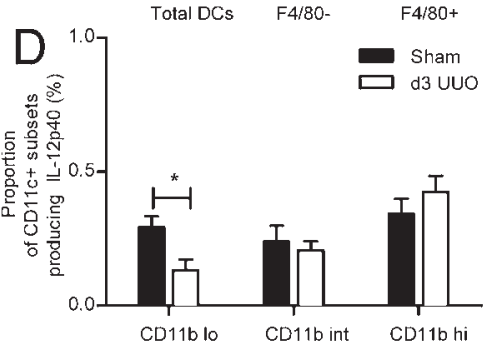

Figure 4. Renal $\mathrm{CD} 11 \mathrm{c}^{+} \mathrm{F} 4 / 80^{-}$cells produce more cytokines after UUO. At day 3 after UUO, TNF, and IL-12p 40 production was compared in kidneys from mice undergoing UUO or sham operation ( $n=5$ /group). The proportion of all CD11 ${ }^{+}$cells producing either TNF (A) or IL-12p40 (B) was similar, but a higher proportion of $\mathrm{CD} 11 \mathrm{c}^{+} \mathrm{F} 4 / 80^{-}$cells produced either cytokine after UUO. There were no consistent and marked changes when $\mathrm{CD} 11 \mathrm{c}^{+} \mathrm{CD} 11 \mathrm{~b}$ cells were examined. A higher proportion of CD $11 b^{\text {lo }}$ and $C D 11 b^{\text {hi }}$ cells made TNF $(\mathbf{C})$, and a lower proportion of $\mathrm{CD}_{11} \mathrm{~b}^{\text {lo }}$ cells made IL-12p40 (D) (although proportions were low for IL12p40). ${ }^{*} P<0.05 ;{ }^{* * *} P<0.01$. collagen deposition, tubular apoptosis, and $\alpha$-SMA expression-show that renal DCs do not alter the early renal fibrosis.

To determine whether depletion of DCs at a later time point alters the development of later fibrosis, CD11c-DTR mice were given DT 7 days after UUO, and injury was studied at day 14 (Figure 10A). Collagen deposition, the accumulation of $\alpha$-SMA + myofibroblasts and numbers of cleaved caspase-3+ apoptotic cells were similar in mice in which DCs had not been depleted and in mice in which DCs were depleted at day 7 (Figure 10, B-F).

\section{Discussion}

The current studies use a suite of in vivo and ex vivo techniques to define the phenotype and role of DCs in innate renal inflammation that results in fibrosis. The well-characterized murine model of ureteric obstruction was used to define the role of DCs in inflammatory events in the kidney. In this model, the cellular and pathological events are similar to those in human disease. ${ }^{29}$ By 3 days after obstruction, leukocyte infiltrates are present in the obstructed kidney, ${ }^{6}$ and overt fibrosis occurs after 7 days, with the deposition of extracellular matrix, including collagens, tubular apoptosis, and myofibroblast activation. ${ }^{30,31}$ In this model, there is no clear evidence of the involvement of adaptive immune responses. ${ }^{12,13}$ The key new findings in the current studies include the following: i) in vivo microscopic evidence of renal DC activation after obstruction; ii) changes in cell surface DC phenotype, reflecting increased activation and recruitment; iii) increased capacity of DCs to activate antigenspecific $\mathrm{CD}^{+} \mathrm{T}$ cells (potentially relevant in allogeneic and infection-triggered responses associated with obstruction); and iv) no direct profibrotic effects for DCs. These results clearly show that renal DCs were activated after UUO with changes in cellular architecture, cell surface markers, and increased antigen-presenting capacity. Despite these phenotypic and functional changes, renal DCs did not aggravate apoptosis or fibrosis.

Multiphoton confocal in vivo microscopy was used to visualize renal DC behavior in real-time in disease for the first time. Live imaging of renal DCs in the normal kidney showed some probing of dendrites from otherwise stationary DCs, consistent with the previous study using this technique. The previous study used CX3CR1-GFP mice, in

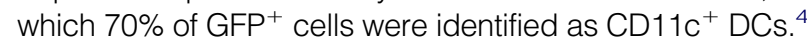
Our studies used mice expressing EYFP under the control of the CD11c promoter, so that at least $95 \%$ of renal EYFP ${ }^{+}$ cells were DCs, providing increased specificity compared with the previous study. This study further extended these findings and provides the very first intravital images of renal DCs under disease conditions. Our studies revealed behavioral changes in DCs during inflammation, including increased cellular activity and an increased number of dendrites extending from the cells, consistent with the elevated activation status of these potent APCs.

The numbers of monocytes, neutrophils, and CD8 T cells were increased in obstructed kidneys 3 days postoperatively. In this respect our findings were similar to those of Dong et al (2008); however, the current study did not find significant increases in the number of DCs in obstructed kidneys. ${ }^{6}$ Absolute cell numbers in the current studies were determined by analyzing samples with a known number of calibration beads, added to whole digested kidneys. This allows accurate assessment of leukocyte numbers between normal and diseased states. It also controls for potential alterations in leukocyte isolation between normal (or sham operated) kidneys and UUO, as damaged tubular cells may not survive the isolation process and injured tubules may be more readily filtered out.

Significant differences in DC surface markers were observed after UUO. Monocyte-derived inflammatory APCs exist in inflammatory lesions, ${ }^{32}$ and phenotypic analysis of renal CD11c + cells after obstruction provided evidence for the recruitment of immature, CD11b $\mathrm{b}^{\text {hi }} \mathrm{Gr}-1^{+}$monocyte-derived inflammatory DCs with lower levels of CD11c migrating into the obstructed kidney. These finding support links between recruited monocytes, macrophages, and DCs, at least in terms of cell surface marker changes. Maturational changes were also identified on resident renal DCs, with loss of F4/80 expression and increased CD11b expression, 
A
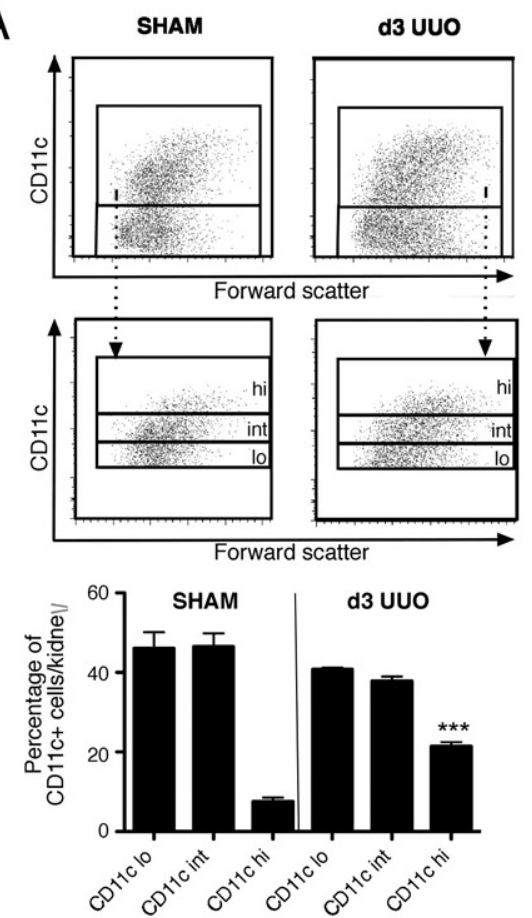

B

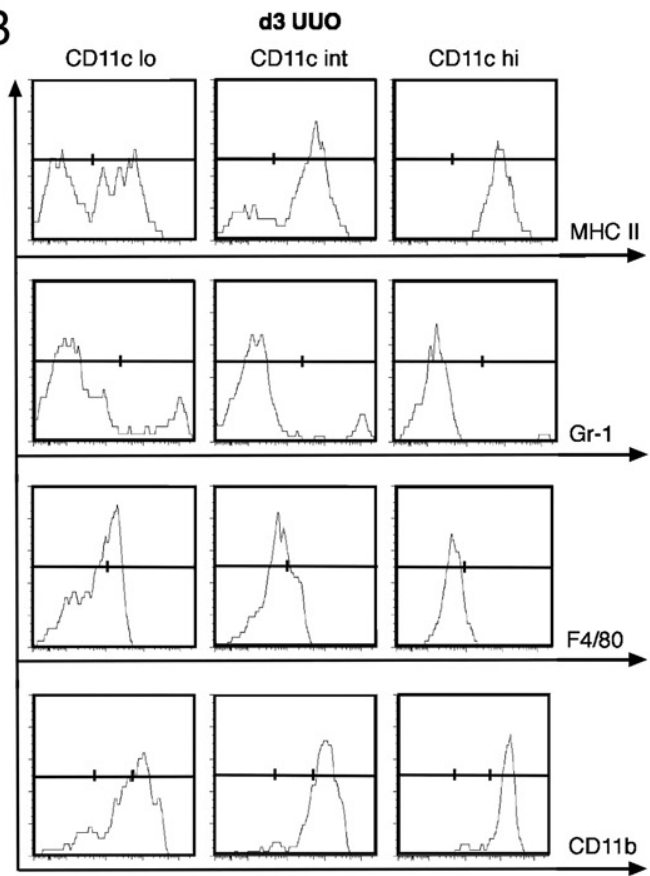

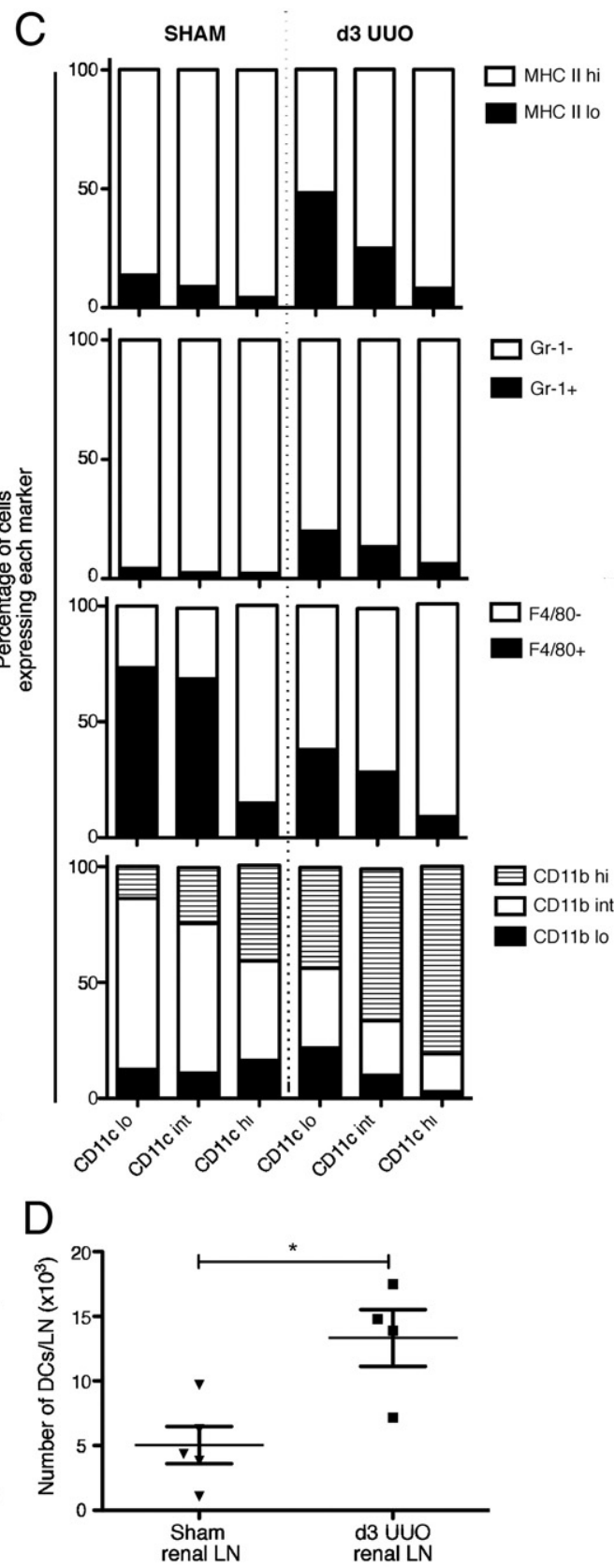

Figure 5. The expression level of CD11c relates to differential expression of markers overlapping with the monocyte/macrophage lineage. Renal CD11 ${ }^{+}$subsets were assessed by multicolor flow-cytometric analysis of single-cell kidney suspensions from mice at d3 UUO or sham-operated controls $(n=5 /$ group). A: Representative dot plots indicate the gating strategy used to identify CD11 ${ }^{+}$expression: CD $11 \mathrm{c}^{+}$cells were further subdivided into three groups based on the surface expression of CD11c being low, intermediate or high (lo, int, or hi). Bar graph indicates the proportion of CD11 ${ }^{+}$cells expressing the differential levels of CD11c for each group. B: Subdivided based on the level of CD11c expression, the proportion of each subset expressing MHC II, Gr-1, F4/80, or CD11b are compared between sham and d3 UUO. C: Representative histograms from a d3 UUO kidney shows the differential expression of MHC II, Gr-1, F4/80, and CD11b on cells that express CD11c at lo, int, or hi levels. D: The absolute number of DCs in the draining renal LN of sham operated and d3 UUO mice ( $n=4 /$ group). ${ }^{*} P<0.05 ;{ }^{* * *} P<0.001$ (d3 UUO versus sham, proportion of CD11c hi DCs).

consistent with their measured increase in function (antigen-specific T-cell responses). In addition, a higher proportion of F4/80- DCs made TNF after UUO, in contrast to that reported by Dong et al, ${ }^{6}$ who found that, 24 hours after UUO, $\mathrm{F} 4 / 80^{+}$cells were the major source of TNF. Although this study demonstrates inflammatory $\mathrm{CD}_{11 \mathrm{c}^{+}}$cells in the injured kidney, further experiments are required to conclusively differentiate between resident and immigrating DCs, to discern their relative contributions in the inflammatory process and to better define the plasticity that exists be- 


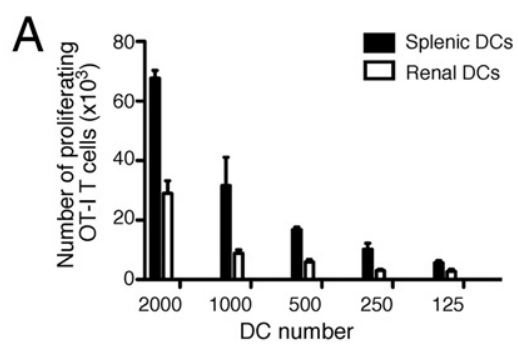

C

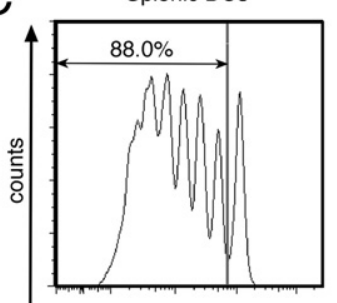

Renal DCs

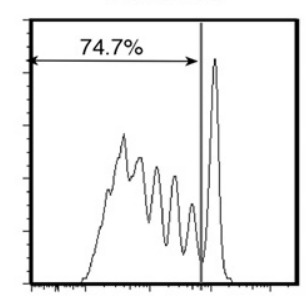

CFSE
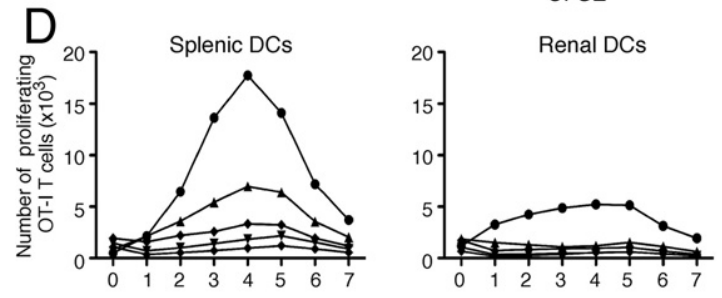

E

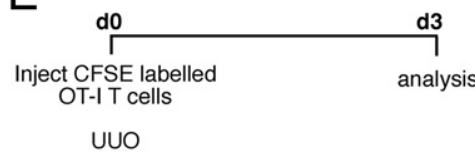

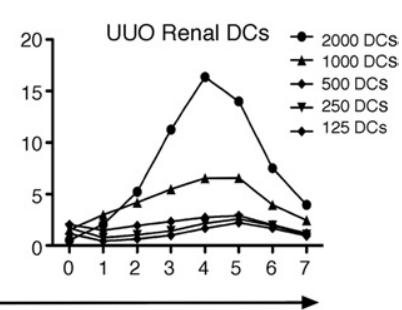

B

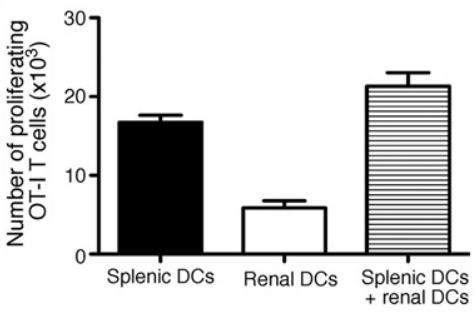

UUO Renal DCs
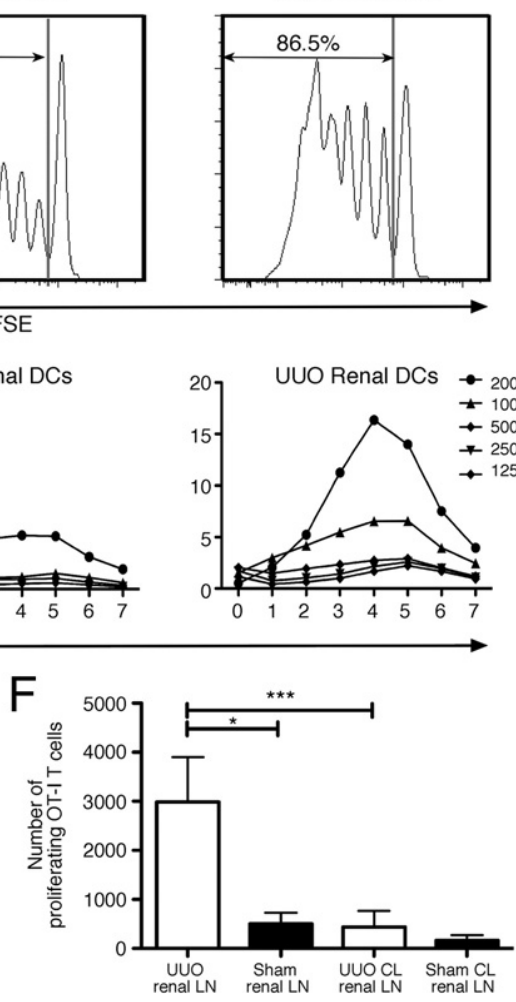

Figure 6. A limited capacity of renal DCs to stimulate T-cell responses is enhanced after UUO. The ability of splenic versus kidney derived DCs to stimulate T-cell responses was tested ex vivo. DCs were isolated from the spleen or kidney and pulsed with OVA peptide before culture with naïve CFSE-labeled OT-I CD8 T cells for 60 hours. A: Comparison of the number of proliferating OT-I T cells (CD8+V $\alpha 2+)$ when stimulated by titrating numbers of splenic versus renal DCs. B: Proliferative T-cell response when cultured with 500 splenic versus 500 renal DCs and the effect of stimulating $\mathrm{T}$ cells with equal numbers of both groups of DCs (500 splenic plus 500 renal DCs). Results of $\mathbf{A}$ and $\mathbf{B}$ are expressec as the mean \pm SEM of triplicate wells. C: Representative histogram profiles and the percentage of OT-I T cells that proliferated in response to stimulation by splenic versus renal versus $d 3$ UUO renal DCs. D: Gives the mean number of proliferating OT-I T cells that have undergone 0 to 7 divisions when stimulated by splenic or renal DCs from healthy donors versus renal DCs from a d3 UUO kidney. Each well was performed in triplicate. E: Shows the experimental protocol used to measure the in vivo proliferation of antigen-specific OT-I T cells. RIPmOVA mice were injected i.v. with CFSE-labeled lymphocytes from OT-I donors. After transfer, the left ureter of recipient mice was surgically ligated (UUO, $n=6$ ), and control mice underwent sham operation $(n=6)$. At day 3 after surgery, mice were sacrificed and the renal lymph nodes (UUO and CL) were collected for flow-cytometric analysis. F: Proliferating OT-I T cells were identified by the dilution of CFSE, and the absolute number of proliferating OT-I T cells per lymph node was quantitated by the incorporation of a known number of Calibrate microbeads per sample. ${ }^{*} P<0.05 ;{ }^{* * * *} P<0.001$ tween monocytes/macrophages and DCs and the nature of the turnover that exists in these cells within the kidney.

Antigen-specific activation of $\mathrm{T}$ cells is the fundamental function of DCs. Renal DCs from noninflamed kidneys were less efficient than splenic DCs in inducing antigen-specific proliferative syngeneic OVA-specific CD8 ${ }^{+}$(OT-I) T cells, consistent with findings in an allogeneic system. ${ }^{3}$ Adding renal DCs to splenic DCs did not inhibit T-cell proliferation. After UUO, a combination of ex vivo and in vivo approaches showed a marked increase in the capacity of DCs to induce antigen-specific T-cell proliferation. Ex vivo data showed that renal DCs were capable of inducing proliferation to levels similar to those caused by splenic DCs, although in vivo studies using CFSE-labeled naive OT-I T cells transferred into syngeneic RIP-mOVA mice (expressing membrane-bound OVA on renal tubules) showed a marked increase in antigen-specific cells in the draining lymph node after UUO. Therefore, after inflammation, renal DCs become more "professional" in their primary function, namely, that of activating antigen-specific T cells. These findings have implications for our understanding of allogeneic and protective immune responses, particularly as urinary tract obstruc- tion can complicate renal transplantation, and urinary tract infection may occur in the presence of obstruction.

We used CD11c-DTR mice to define the roles of DCs in inflammatory renal disease leading to fibrosis. DC depletion studies in glomerulonephritis induced by an adaptive immune responses to a foreign antigen show that initially, DCs were protective ${ }^{7}$ but later in disease acquired a pathogenic role, ${ }^{8}$ whereas in cisplatin-induced nephrotoxicity, DCs are protective. ${ }^{9}$ Renal DCs are sources of TNF which can induce renal epithelial cell apoptosis, ${ }^{6}$ and TNF neutralization attenuates both apoptosis and fibrosis. ${ }^{33,34}$ In the current studies, there was no overall increase in the proportion of renal DCs with the capacity to make TNF, although increased proportions of the $\mathrm{CD} 11 \mathrm{C}^{+} \mathrm{F} 4 / 80^{-}$subset produced TNF by day 3. However, no difference was found in key indices of injury after DC depletion, regardless of the time point at which that DCs were ablated. The extent of collagen accumulation, the number of apoptotic cells and the expression of $\alpha$-SMA were similar between groups. Although $\mathrm{CD}_{11 \mathrm{c}^{+}}$cell depletion is not complete in the DTR model, studies in other experimental renal diseases have found an effect after DC depletion, ${ }^{7-9}$ indicating the degree 

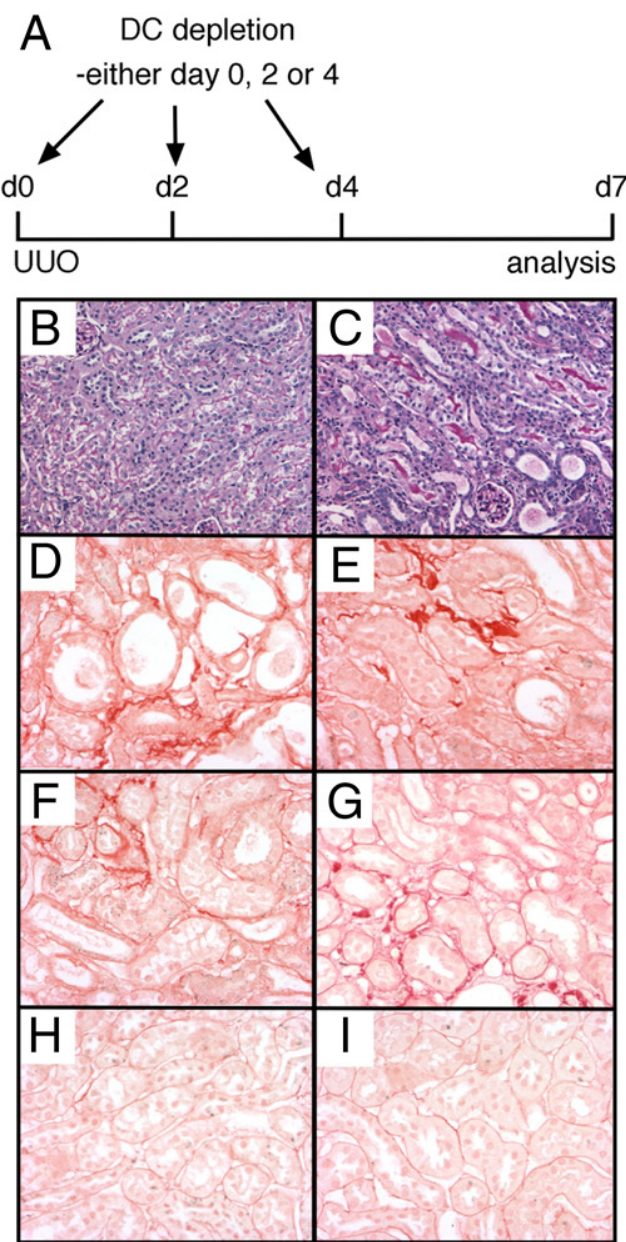

$\mathrm{J}$

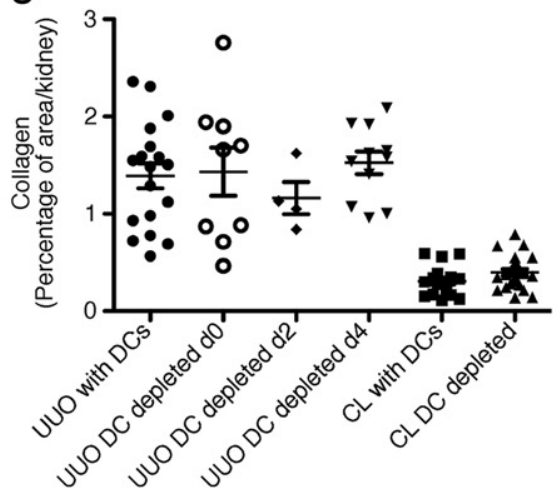

Figure 7. DC depletion at different time points does not attenuate collagen deposition in the obstructed kidney. A: Experimental protocol used to establish the role of DCs in the progression of kidney fibrosis. The left kidney of CD11CDTR mice (transgene negative or wild-type mice as controls) was obstructed on day 0. At day 0,2 or 4 , diphtheria toxin was administered to deplete DCs, and at day 7 kidneys were collected for histological analysis. Both the UUO and CL kidneys were collected for histological analysis. Representative photomicrographs of periodic acidSchiff-stained kidney sections of a CL kidney (B) and an obstructed kidney at day 7 (C). Original magnification, $\times 200$. Representative photomicrographs showing picrosirius red staining for collagen fibers are shown. UUO kidney of a control mouse (D) mouse depleted of DCs at day $0(\mathbf{E})$, mouse depleted of DCs at day 2 (F), mouse depleted of DCs at day $4(\mathbf{G})$, and CL kidneys of a control mouse (H) and a mouse depleted of DCs (I). J: Results are expressed as the percentage area of the kidney comprising collagen (mean taken from five to 10 nonoverlapping fields of view); magnification, $\times 400$. Depletion of DCs at each time point was performed as a separate experiment with nondepleted controls. For presentation purposes, results are collated on one graph where controls (UUO with DCs, CL with DCs, and CL DC depleted) are combined from the individual experiments. Statistical analysis compared each experimental group to the control group from the same experiment. Data points represent individual mice.
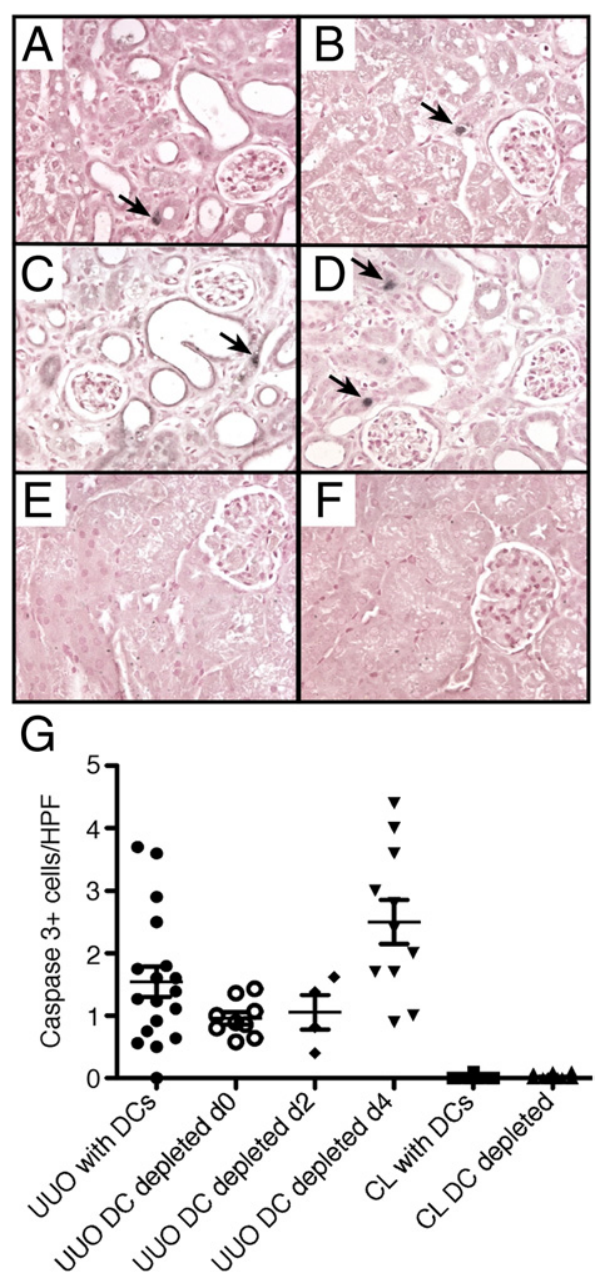

Figure 8. DC depletion does not reduce the number of apoptosing cells in the obstructed kidney. The experimental protocol used to establish the role of DCs in the progression of kidney fibrosis is outlined in Figure 6A. The same kidney samples were stained with an anticleaved caspase- $3 \mathrm{Ab}$ to enumerate the number of apoptosing cells per kidney. Representative photomicrographs showing caspase-3 staining. UUO kidney of a control mouse (A), mouse depleted of DCs at day 0 (B) mouse depleted of DCs at day 2 (C), mouse depleted of DCs at day 4 (D) and the CL kidney of a control mouse (E) and a mouse depleted of DCs (F). G: The number of cells that stained for caspase- 3 was manually counted (mean taken from 10 to 15 nonoverlapping high-power fields [HPF] of view; magnification, $\times 400$ ). For presentation purposes, results are collated on one graph in which controls (UUO with DCs, CL with DCs, and CL DC depleted) are combined from the individual experiments. Statistical analysis compared each experimental group to the control group from the same experiment. Data points represent individual mice.

of depletion achieved by this model is biologically relevant. Nonetheless, it is possible that the remaining DCs in UUO may play a role in this condition. Plasmacytoid DCs, a minor population of renal DCs, may or may not be effectively depleted by DT, ${ }^{35,36}$ and although they traditionally play a role in antiviral responses, it remains possible that pDCs may directly influence fibrosis. Our study primarily investigated the role of DCs in the early inflammatory events. Although we also examined the effect of DC depletion at day 7 on outcomes at day 14, it remains possible that DCs may play a significant functional role at a late stage of fibrosis.

Previous studies that have used less specific depletion studies have also affected renal DCs. One used liposomal clodronate to deplete phagocytic cells (including F4/80+ 

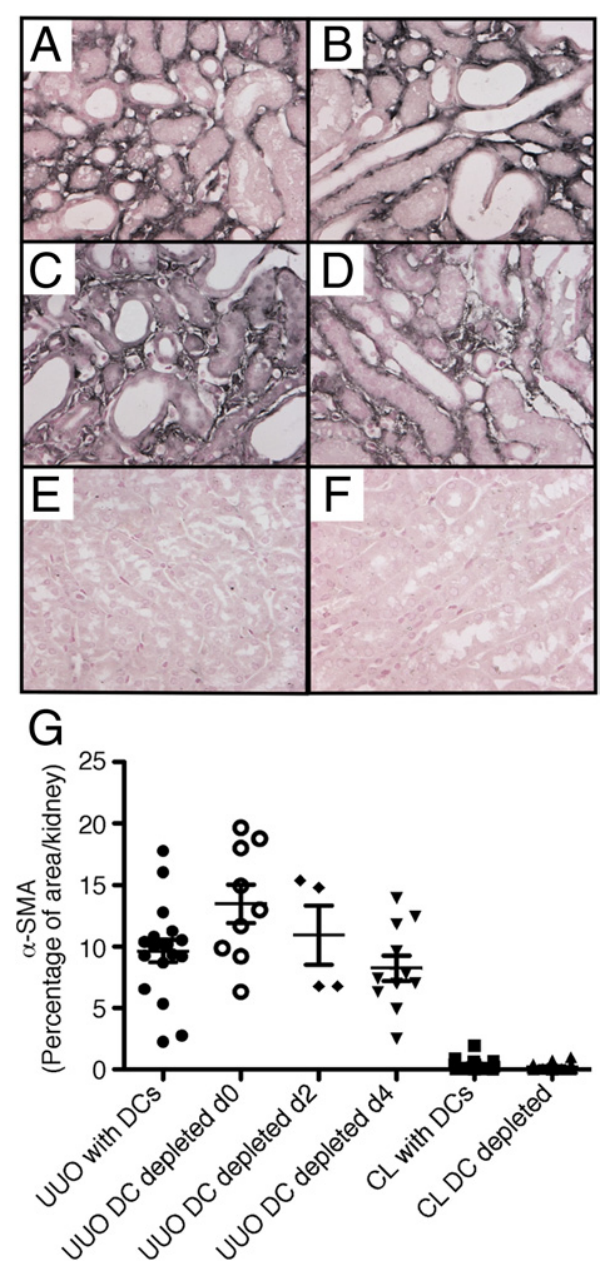

Figure 9. Myofibroblast formation is not inhibited by the depletion of DCs during UUO. The experimental protocol used to establish the role of DCs in the progression of kidney fibrosis is outlined in Figure 7A. Kidney samples were also stained with anti- $\alpha$ SMA a marker for myofibroblasts. Representative photomicrographs showing $\alpha \mathrm{SMA}^{+}$staining. UUO kidney of a control mouse (A), mouse depleted of DCs at day $0(\mathbf{B})$, mouse depleted of DCs at day $2(\mathbf{C})$, mouse depleted of DCs at day $4(\mathbf{D})$, and CL kidney of a control mouse (E) and a mouse depleted of DCs (F). G: Results are expressed as the percentage area of the kidney comprising $\alpha$-SMA as determined using MetaMorph (mean taken from five to 10 nonoverlapping fields of view; magnification $\times 400$ ). For presentation purposes, results are collated on one graph in which controls (UUO with DCs, CL with DCs, and CL DC depleted) are combined from the individual experiments. Statistical analysis compared each experimental group to the control group from the same experiment. Data points represent individual mice.

macrophages and DCs, and potentially neutrophils) after UUO, showing attenuated fibrosis and suggested a collective pro-inflammatory role for phagocytes. ${ }^{37}$ Similar results are reported in studies that conditionally ablated CD11bexpressing cells, ${ }^{38,39}$ which include renal macrophages and some DCs. However, these studies could not address a role for DCs in the progression of fibrosis, distinct from the contributions of macrophages (and potentially neutrophils). Therefore, the results of the current studies implicate macrophages more directly in the pathogenesis of renal fibrosis, although the diverse phenotype of tissue macrophages implies divergent roles for different macrophage subsets that may be time dependent. Most recently, CD11C-DTR chimeric mice (to allow repeated administration of DT) were de- pleted of DCs ${ }^{17,39}$ before a 5-day model of UUO, with no effect on collagen accumulation. ${ }^{39}$

In summary, in the context of interstitial inflammation, renal DCs are activated and function as more potent APCs and better stimulate T-cell responses. Their pro-inflammatory functions are related largely to their classical role as activators of T cells, and they do not directly contribute to
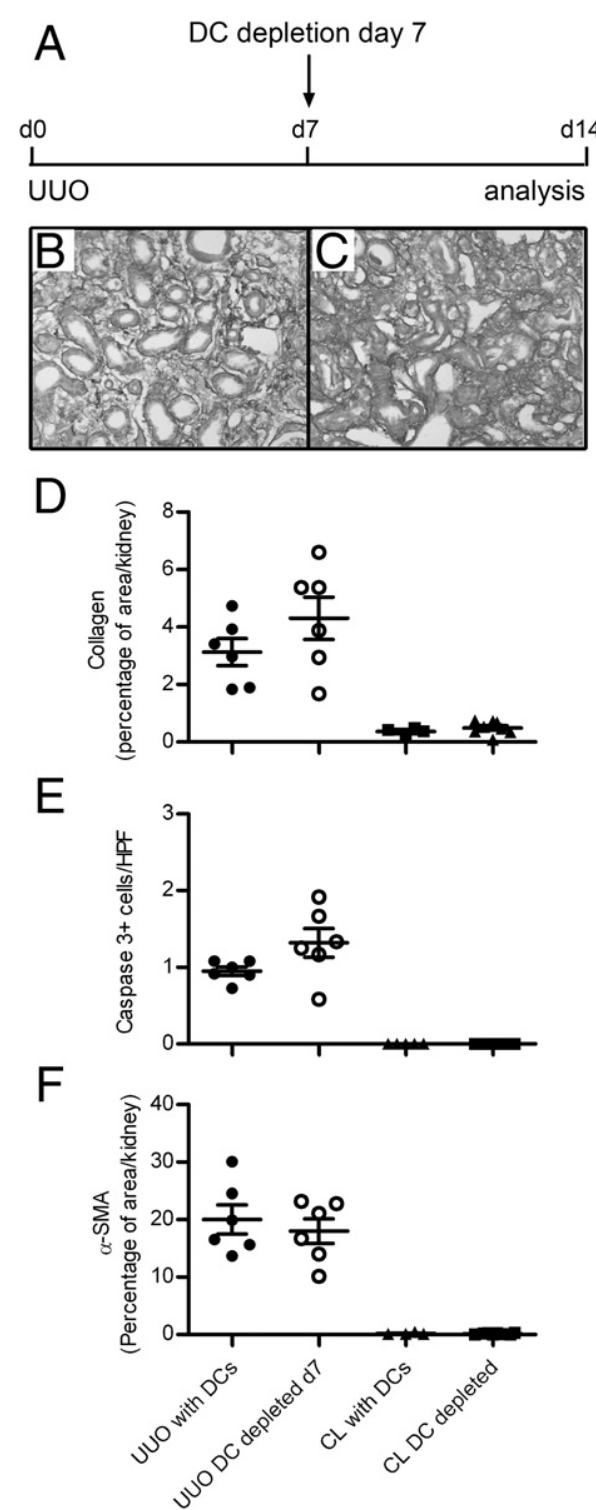

Figure 10. The effect of DC depletion 7 days after UUO on collagen deposition, myofibroblast accumulation, and apoptosis at day 14. A: Experimental protocol used to establish the role of DCs in the progression of kidney fibrosis. The left kidney of CD11cDTR mice $(n=6$, transgene negative or wild-type mice as controls, $n=6$ ) was obstructed on day 0. At day 7 , diphtheria toxin was administered to deplete the DCs and kidneys were collected for histological analysis on day 14. Both the UUO and CL kidneys were collected for histological analysis. Representative photomicrographs showing picrosirius red staining for collagen fibers are shown, with (B) a UUO kidney of a control mouse at day 14 and (C) a mouse depleted of DCs at day 7 , studied at day 14 . There was no significant difference in collagen accumulation (D), the number of cleaved-caspase- $3^{+}$apoptotic cells, expressed as cells per high-power field (HPF; E) or myofibroblast accumulation (F) after DC depletion. Results are expressed as the percentage area of the kidney comprising collagen or $\alpha$-SMA (mean taken from five to 10 nonoverlapping fields of view; magnification $\times 400$ ) and for caspase- 3 the number of positive cells in $>10$ fields. Data points represent individual mice. 
fibrosis or renal cell apoptosis in the early phases of renal fibrosis.

\section{Acknowledgments}

We thank Alice Wright and Chrystel Llanto for technical assistance and James Ngui and Dale Baum for support from the Flow cytometry Facility (Department of Medicine, Monash Medical Centre). We also appreciate the assistance of staff at Monash Micro Imaging, Monash Medical Centre Animal Facilities, and the House for Experimental Therapy.

\section{References}

1. Hart DN, Fabre JW: Demonstration and characterization of la-positive dendritic cells in the interstitial connective tissues of rat heart and other tissues, but not brain. J Exp Med 1981, 154:347-361

2. Austyn JM, Hankins DF, Larsen CP, Morris PJ, Rao AS, Roake JA: Isolation and characterization of dendritic cells from mouse heart and kidney. J Immunol 1994, 152:2401-2410

3. Kruger T, Benke D, Eitner F, Lang A, Wirtz M, Hamilton-Williams EE, Engel D, Giese B, Muller-Newen G, Floege J, Kurts C: Identification and functional characterization of dendritic cells in the healthy murine kidney and in experimental glomerulonephritis. J Am Soc Nephrol 2004, 15:613-621

4. Soos TJ, Sims TN, Barisoni L, Lin K, Littman DR, Dustin ML, Nelson PJ: CX3CR1 + interstitial dendritic cells form a contiguous network throughout the entire kidney. Kidney Int 2006, 70:591-596

5. Dong X, Swaminathan S, Bachman LA, Croatt AJ, Nath KA, Griffin MD: Resident dendritic cells are the predominant TNF-secreting cell in early renal ischemia-reperfusion injury. Kidney Int 2007, 71:619-628

6. Dong X, Bachman LA, Miller MN, Nath KA, Griffin MD: Dendritic cells facilitate accumulation of IL-17 T cells in the kidney following acute renal obstruction. Kidney Int 2008, 74:1294-1309

7. Scholz J, Lukacs-Kornek V, Engel DR, Specht S, Kiss E, Eitner F, Floege J, Groene HJ, Kurts C: Renal dendritic cells stimulate IL-10 production and attenuate nephrotoxic nephritis. J Am Soc Nephrol 2008, 19:527-537

8. Hochheiser K, Engel DR, Hammerich L, Heymann F, Knolle PA, Panzer U, Kurts C: Kidney dendritic cells become pathogenic during crescentic glomerulonephritis with proteinuria. J Am Soc Nephrol 2011, 22:306-316

9. Tadagavadi RK, Reeves WB: Renal dendritic cells ameliorate nephrotoxic acute kidney injury. J Am Soc Nephrol 2010, 21:53-63

10. Klahr S, Morrissey JJ: The role of growth factors, cytokines, and vasoactive compounds in obstructive nephropathy. Semin Nephrol 1998, 18:622-632

11. Misseri R, Rink RC, Meldrum DR, Meldrum KK: Inflammatory mediators and growth factors in obstructive renal injury. J Surg Res 2004, 119:149-159

12. Tapmeier TT, Fearn A, Brown K, Chowdhury P, Sacks SH, Sheerin NS, Wong W: Pivotal role of CD4+ T cells in renal fibrosis following ureteric obstruction. Kidney Int 78:351-362

13. Shappell SB, Gurpinar T, Lechago J, Suki WN, Truong LD: Chronic obstructive uropathy in severe combined immunodeficient (SCID) mice: lymphocyte infiltration is not required for progressive tubulointerstitial injury. J Am Soc Nephrol 1998, 9:1008-1017

14. Clarke SR, Barnden M, Kurts C, Carbone FR, Miller JF, Heath WR: Characterization of the ovalbumin-specific TCR transgenic line OT-I: $\mathrm{mHC}$ elements for positive and negative selection. Immunol Cell Biol 2000, 78:110-117

15. Lindquist RL, Shakhar G, Dudziak D, Wardemann H, Eisenreich T, Dustin ML, Nussenzweig MC: Visualizing dendritic cell networks in vivo. Nat Immunol 2004, 5:1243-1250

16. Kurts C, Heath WR, Carbone FR, Allison J, Miller JF, Kosaka H: Constitutive class I-restricted exogenous presentation of self antigens in vivo. J Exp Med 1996, 184:923-930

17. Jung S, Unutmaz D, Wong P, Sano G, De los Santos K, Sparwasser T, Wu S, Vuthoori S, Ko K, Zavala F, Pamer EG, Littman DR, Lang RA: In vivo depletion of CD11c + dendritic cells abrogates priming of CD8 $+\mathrm{T}$ cells by exogenous cell-associated antigens. Immunity 2002, 17:211-220
18. Kuligowski MP, Kitching AR, Hickey MJ: Leukocyte recruitment to the inflamed glomerulus: a critical role for platelet-derived P-selectin in the absence of rolling. J Immunol 2006, 176:6991-6999

19. Wadell H: Volume. Shape, and roundness of quartz particles. J Geol 1935, 43:250-280

20. Roediger B, Ng LG, Smith AL, Fazekas de St Groth B, Weninger W: Visualizing dendritic cell migration within the skin. Histochem Cell Biol 2008, 130:1131-1146

21. van den Berg TK, Kraal G: A function for the macrophage F4/80 molecule in tolerance induction. Trends Immunol 2005, 26:506-509

22. del Hoyo GM, Martin P, Vargas HH, Ruiz S, Arias CF, Ardavin C: Characterization of a common precursor population for dendritic cells. Nature 2002, 415:1043-1047

23. Randolph GJ, Inaba K, Robbiani DF, Steinman RM, Muller WA: Differentiation of phagocytic monocytes into lymph node dendritic cells in vivo. Immunity 1999, 11:753-761

24. Varol C, Landsman L, Fogg DK, Greenshtein L, Gildor B, Margalit R, Kalchenko V, Geissmann F, Jung S: Monocytes give rise to mucosal, but not splenic, conventional dendritic cells. J Exp Med 2007, 204:171-180

25. Ferenbach D, Hughes J: Macrophages and dendritic cells: what is the difference? Kidney Int 2008, 74:5-7

26. O'Keeffe M, Hochrein H, Vremec D, Scott B, Hertzog P, Tatarczuch L, Shortman K: Dendritic cell precursor populations of mouse blood: identification of the murine homologues of human blood plasmacytoid pre-DC2 and CD11c+ DC1 precursors. Blood 2003, 101:1453-1459

27. Randolph GJ, Angeli V, Swartz MA: Dendritic-cell trafficking to Iymph nodes through lymphatic vessels. Nat Rev Immunol 2005, 5:617-628

28. Lin HH, Faunce DE, Stacey M, Terajewicz A, Nakamura T, ZhangHoover J, Kerley M, Mucenski ML, Gordon S, Stein-Streilein J: The macrophage $\mathrm{F} 4 / 80$ receptor is required for the induction of antigenspecific efferent regulatory $T$ cells in peripheral tolerance. J Exp Med 2005, 201:1615-1625

29. Chevalier RL, Forbes MS, Thornhill BA: Ureteral obstruction as a model of renal interstitial fibrosis and obstructive nephropathy. Kidney Int 2009, 75:1145-1152

30. Bascands JL, Schanstra JP: Obstructive nephropathy: insights from genetically engineered animals. Kidney Int 2005, 68:925-937

31. Liu Y: Renal fibrosis: new insights into the pathogenesis and therapeutics. Kidney Int 2006, 69:213-217

32. Dominguez PM, Ardavin C: Differentiation and function of mouse monocyte-derived dendritic cells in steady state and inflammation. Immunol Rev 2010, 234:90-104

33. Meldrum KK, Metcalfe P, Leslie JA, Misseri R, Hile KL, Meldrum DR: TNF-alpha neutralization decreases nuclear factor-kappaB activation and apoptosis during renal obstruction. J Surg Res 2006, 131:182-188

34. Meldrum KK, Misseri R, Metcalfe P, Dinarello CA, Hile KL, Meldrum DR: TNF-alpha neutralization ameliorates obstruction-induced renal fibrosis and dysfunction. Am J Physiol Regul Integr Comp Physiol 2007, 292:R1456-R1464

35. Kassim SH, Rajasagi NK, Zhao X, Chervenak R, Jennings SR: In vivo ablation of CD11c-positive dendritic cells increases susceptibility to herpes simplex virus type 1 infection and diminishes NK and T-cell responses. J Virol 2006, 80:3985-3993

36. Sapoznikov A, Fischer JA, Zaft T, Krauthgamer R, Dzionek A, Jung S: Organ-dependent in vivo priming of naive CD4+, but not CD8+. T cells by plasmacytoid dendritic cells. J Exp Med 2007, 204: 1923-1933

37. Kitamoto K, Machida Y, Uchida J, Izumi Y, Shiota M, Nakao T, Iwao H, Yukimura T, Nakatani T, Miura K: Effects of liposome clodronate on renal leukocyte populations and renal fibrosis in murine obstructive nephropathy. J Pharmacol Sci 2009, 111:285-292

38. Lin SL, Castano AP, Nowlin BT, Lupher ML, Jr., Duffield JS: Bone marrow Ly6Chigh monocytes are selectively recruited to injured kidney and differentiate into functionally distinct populations. J Immunol 2009, 183:6733-6743

39. Machida Y, Kitamoto K, Izumi Y, Shiota M, Uchida J, Kira Y, Nakatani $\mathrm{T}$, Miura K: Renal fibrosis in murine obstructive nephropathy is attenuated by depletion of monocyte lineage, not dendritic cells. J Pharmacol Sci 2010, 114:464-473 